\title{
Enhanced Light Harvesting in Dye-Sensitized Solar Cell Using External Lightguide
}

\author{
Chi-Hui Chien, ${ }^{1}$ Ming-Lang Tsai, ${ }^{1}$ Chi-Chang Hsieh, ${ }^{2}$ and Yan-Huei Li ${ }^{2}$ \\ ${ }^{1}$ Department of Mechanical and Electro-Mechanical Engineering, National Sun Yat-Sen University, Kaohsiung 804, Taiwan \\ ${ }^{2}$ Department of Mechanical and Automation Engineering, National Kaohsiung First University of Science and Technology, \\ Kaohsiung 811, Taiwan
}

Correspondence should be addressed to Chi-Hui Chien, chchien@faculty.nsysu.edu.tw

Received 28 March 2011; Accepted 6 July 2011

Academic Editor: Abderrazek Douhal

Copyright () 2011 Chi-Hui Chien et al. This is an open access article distributed under the Creative Commons Attribution License, which permits unrestricted use, distribution, and reproduction in any medium, provided the original work is properly cited.

\begin{abstract}
An external lightguide (EL) for enhancing the light-harvesting efficiency of dye-sensitized solar cells (DSSCs) was designed and developed. The EL attached to the exterior of a DSSC photoelectrode directed light on a dye-covered nanoporous $\mathrm{TiO}_{2}$ film $(\mathrm{D}-$ NTF) of the photoelectrode. Experimental tests confirmed that the EL increased the light-harvesting efficiency of a DSSC with an active area of $0.25 \mathrm{~cm}^{2}$ by $30.69 \%$. Photocurrent density and the power conversion efficiency were also increased by $38.12 \%$ and $25.09 \%$, respectively.
\end{abstract}

\section{Introduction}

Dye-sensitized solar cells (DSSCs) have been extensively studied in recent years [1-3], owing to their potentially cost-effective and easy mass production characteristics. A DSSC comprises four key components, which are the photoelectrode $[4,5]$, a platinum $(\mathrm{Pt})$ counterelectrode $[6,7]$ deposited opposite the photoelectrode, an electrolyte solution $[5,6]$ containing an iodide/tri-iodide redox couple between the photo- and counterelectrodes, and a sealing spacer $[8,9]$ to fix the space between the photo- and counterelectrodes and to seal the electrolyte solution. The photoelectrode designed to support light-to-electricity conversion is normally formed from a dye-covered nanoporous $\mathrm{TiO}_{2}$ film (D-NTF) that is fabricated on a transparent conducting substrate. Excitation of the dye by light illuminating the D-NTF of the photoelectrode causes a rapid injection of electrons into the conduction band of the $\mathrm{TiO}_{2}$ and their further movement toward the transparent conducting substrate. However, the light also penetrates the sealing spacer, eventually exiting the cell. The light illuminating the D-NTF is therefore captured, whereas the light illuminating the sealing spacer is wasted. This phenomenon is evident in a gridtype DSSC $[10,11]$. In the study by Lee et al. [10] and Ramasamy et al. [11], the use of multiple sealing spacers to seal each cell separately resulted in excessive light penetration into the sealing spacers, which rendered them unusable. Restated, the light-harvesting efficiency of the DSSC can be enhanced by directing the light toward the D-NTF before it illuminates the sealing spacer. Therefore, this work proposes a transparent external lightguide (EL) that improves the photovoltaic performance of the DSSC by directing light toward the D-NTF.

\section{EL Design}

Figure 1 shows that, in an illuminated DSSC, some light received by the D-NTF of the photoelectrode (PE) is absorbed by the dye. Some light also penetrates the photoelectrode (PE), the sealing spacers (SS), and the counterelectrode $(\mathrm{CE})$, in that order, before finally exiting the cell. Hence, the light illuminating the sealing spacer was wasted. To obtain a high light-harvesting efficiency, this study used an external lightguide (EL) made of a transparent medium to direct the light toward the D-NTF. To maximize the intensity of light received by the D-NTF, the EL is designed to minimize optical loss along all trajectories of incoming light. Accordingly, the EL exploits both normal incidence and total internal reflection (TIR). The normal incidence 


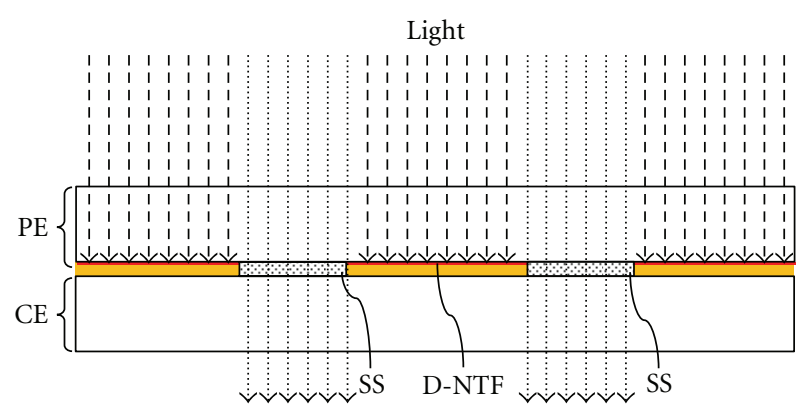

FIGURE 1: Illumination of DSSC.

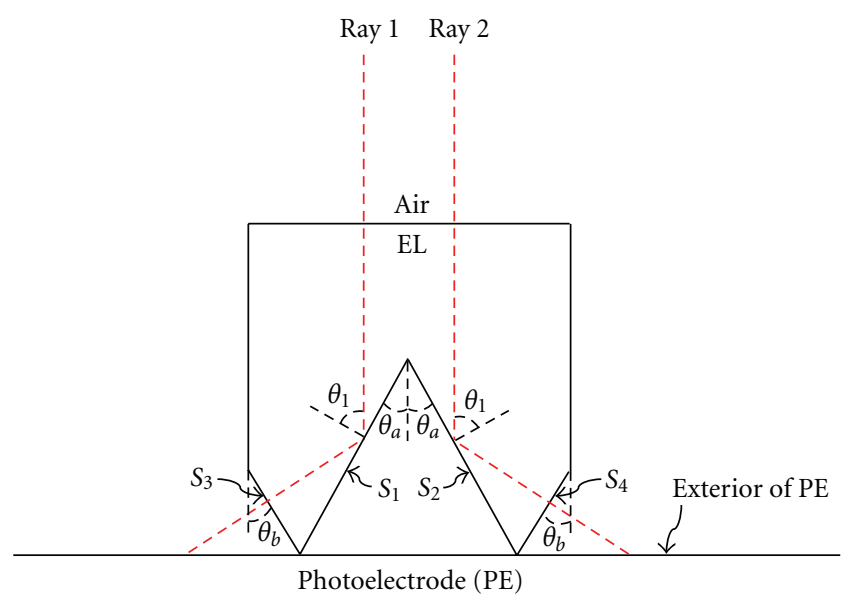

Figure 2: Proposed EL architecture.

condition ensures that the light travels from medium 1 into medium 2 with the lowest possible reflection loss. The TIR condition ensures that the light travels from medium 1 into medium 2 without transmission loss. Figure 2 presents an EL architecture that satisfies both conditions. The figure clearly reveals that the EL has a symmetric prism structure whose geometry is defined by two optical angles $\left(\theta_{a}\right.$ and $\left.\theta_{b}\right)$ and four optical interfaces $\left(S_{1}, S_{2}, S_{3}\right.$, and $\left.S_{4}\right)$. The trajectories of rays 1 and 2 clearly show that this design meets the normal incidence condition as light travels through the air and enters the EL. When the light travels from EL into air across interfaces $S_{1}$ and $S_{2}$, TIR occurs, ensuring an absence of transmission loss. Hence, the incident angle $\theta_{1}$ should satisfy the TIR condition. The $\theta_{1}$ for TIR is obtained by the Snell's law:

$$
\theta_{1}>\sin ^{-1}\left(\frac{1}{n_{\mathrm{EL}}}\right),
$$

where $n_{\mathrm{EL}}$ is the refractive index of the EL. After the light rays are reflected from interfaces $S_{1}$ and $S_{2}$, they are normally incident at interfaces $S_{3}$ and $S_{4}$, respectively, which maximizes transmittance and minimizes reflection loss. Based on the $\theta_{1}$ for TIR and the normal incidence at interfaces $S_{3}$ and $S_{4}$, the optical angles $\theta_{a}$ and $\theta_{b}$, respectively, of the symmetric prism structure are obtained using the following formulae

$$
\theta_{a}=90^{\circ}-\theta_{1},
$$

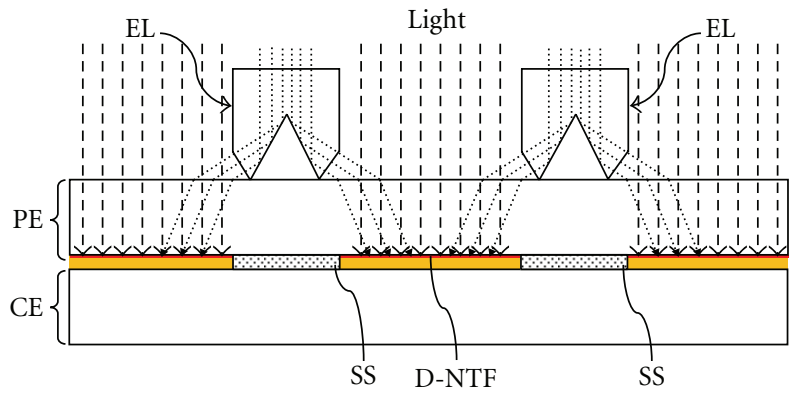

FIgURE 3: Illustration of EL in directing light toward D-NTF.

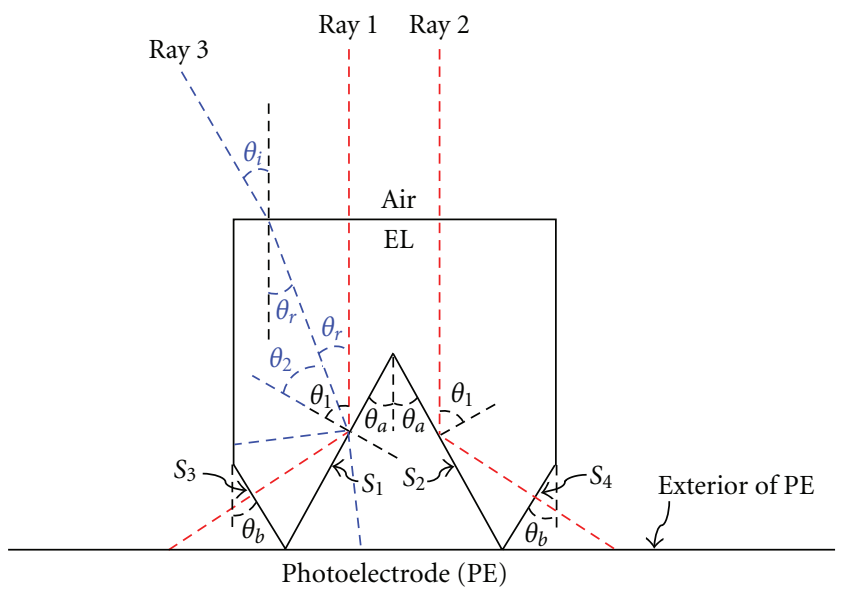

FIGURE 4: Example of oblique illumination of the EL at an incident angle of $\theta_{i}$.

$$
\theta_{b}=2 \theta_{1}-90^{\circ}
$$

According to the above equations, the symmetric prism design depends on $\theta_{1}$. The EL on the exterior of the DSSC photoelectrode effectively directs the light toward the D-NTF (Figure 3). Light arriving at the D-NTF has the highest possible intensity since the transmittance of light traveling from the EL into the air is maximized.

Notably, the above EL is most effective when light is normally incident onto the EL. Figure 4 shows an example of the oblique illumination (by ray 3 ) of the EL at an incident angle of $\theta_{i}$. A transmission loss occurs at interface $S_{1}$ because the incident angle $\theta_{2}$ is smaller than the $\theta_{1}$ for TIR. This phenomenon occurs if $\theta_{i}$ exceeds $0^{\circ}$. To ensure that obliquely incident light can also be directed toward the D-NTF with minimal optical loss, $\theta_{2}$ must also satisfy the TIR condition, which requires modification of $\theta_{1}$. Figure 4 shows that $\theta_{1}$ can be reformulated as

$$
\theta_{1}=\theta_{2}+\theta_{r}
$$

where $\theta_{r}$ is the angle of refraction, which can be obtained by Snell's law:

$$
\theta_{r}=\sin ^{-1}\left(\frac{\sin \theta_{i}}{n_{\mathrm{EL}}}\right) .
$$


TAble 1: Values for $\theta_{1}, \theta_{a}$, and $\theta_{b}$ obtained by equations (2), (3), and (6), respectively.

\begin{tabular}{lccc}
\hline Data & $\theta_{1}$ & $\theta_{a}$ & $\theta_{b}$ \\
\hline Calculated & $61.473^{\circ}$ & $28.527^{\circ}$ & $32.546^{\circ}$ \\
Compensated & $62.473^{\circ}$ & $27.527^{\circ}$ & $34.946^{\circ}$ \\
\hline
\end{tabular}

TABLe 2: Photovoltaic performance of DSSC with and without EL.

\begin{tabular}{lcccc}
\hline DSSC & $J_{\mathrm{sc}}\left(\mathrm{mA} / \mathrm{cm}^{2}\right)$ & $V_{\mathrm{oc}}(\mathrm{V})$ & $\mathrm{FF}$ & $\eta(\%)$ \\
\hline Without EL & 6.90 & 0.685 & 0.591 & 2.79 \\
With one EL & 8.04 & 0.676 & 0.581 & 3.16 \\
With two ELs & 9.53 & 0.687 & 0.533 & 3.49 \\
\hline
\end{tabular}

According to equations (4) and (5) and where $\theta_{2}$ satisfies the TIR condition,

$$
\theta_{1}>\sin ^{-1}\left(\frac{1}{n_{\mathrm{EL}}}\right)+\sin ^{-1}\left(\frac{\sin \theta_{i}}{n_{\mathrm{EL}}}\right) .
$$

According to equations (2) and (6), $\theta_{i}$ increases as $\theta_{1}$ increases and as $\theta_{a}$ decreases. The decrease in $\theta_{a}$ reduces the reflective area at interfaces $S_{1}$ and $S_{2}$ as well as the light-harvesting efficiency. Therefore, $\theta_{i}$ must be maintained at a suitable value. This study sets $\theta_{i}$ to $30^{\circ}$, which illuminated the EL at an incident angle between $0^{\circ}$ and $30^{\circ}$. The resulting TIR at interfaces $S_{1}$ and $S_{2}$ prevented transmission loss. The refractive index $n_{\mathrm{EL}}$ is a function of wavelength; that is, the index is high at short wavelengths and low at long wavelengths. The EL was designed to have a low refractive index at a long wavelength of $800 \mathrm{~nm}$ to ensure that light with wavelength shorter than $800 \mathrm{~nm}$ would undergo TIR at interfaces $S_{1}$ and $S_{2}$.

\section{EL Fabrication}

The EL was fabricated from UV-curable acrylic resin, which cures rapidly and is easily released from the mold. For mass production, however, non-UV-curable acrylic resin would be preferable. Ellipsometer measurements of the cured acrylic resin showed a refractive index of 1.496 at a wavelength of $800 \mathrm{~nm}$. The EL had a width of $3 \mathrm{~mm}$ wide, which was the same as that of the sealing spacer. Table 1 shows the values for $\theta_{1}, \theta_{a}$, and $\theta_{b}$, which were calculated by equations (2), (3), and (6), respectively. Notably, the calculated $\theta_{1}$ is a lowest limiting value. Table 1 also shows how the analysis compensated for errors in machining the EL mold. The calculated $\theta_{1}$ plus a compensation value of $1^{\circ}$, the calculated $\theta_{a}$ minus a compensation value of $1^{\circ}$, and the calculated $\theta_{b}$ plus a compensation value of $2.4^{\circ}$ are referred to as "compensated data." The compensated data were used to design an EL mold $65 \mathrm{~mm}$ wide, $20 \mathrm{~mm}$ high, and $100 \mathrm{~mm}$ long. The two-cavity EL mold was fabricated by a wire-cut machining method, and the mold was polished to a mirror-like surface. Figure 5 is a photograph of the EL mold, which clearly reveals that the mold had a machining error smaller than $0.05^{\circ}$, which was much smaller than the compensation value of $1^{\circ}$.

Acrylic resin was infused into the EL mold, which was then placed in a vacuum chamber to remove the air bubbles from the resin. A glass plate was placed on the top of the EL mold to level the acrylic resin. After the resin cured, the glass plate was removed, and the EL was released from the mold. The EL was divided into several sections (Figure 6) to match the area of the D-NTF.

\section{Measurement of Light-Harvesting Efficiency}

In Figure 3, the EL was used to direct light toward the D-NTF of the photoelectrode. Therefore, the analysis of the light-harvesting efficiency focused on the D-NTF, and the experimental model of the DSSC that was utilized in measuring the light-harvesting efficiency was simplified by including only the photoelectrode. The experimental system that is presented in Figure 7 was established to investigate the light-harvesting efficiency of the photoelectrode with the ELs. The experimental system comprised (i) a solar power meter, (ii) a solar simulator, (iii) an FTO glass substrate, and (iv) ELs. The solar power meter measured the solar light intensity, and its light-absorbing detector (area: $0.5 \times$ $0.5 \mathrm{~cm}^{2}$ ) could be used as a D-NTF. The solar simulator, which was capable of simulating AM 1.5 solar radiation, was adjusted to $1000 \mathrm{~W} / \mathrm{m}^{2}$, which is equivalent to one sun illumination. The FTO glass substrate deposited on the solar power detector served as the photoelectrode. The ELs were then arranged on the FTO glass substrate to investigate the variations in light intensity displays by using the solar power meter. The light-harvesting efficiency of the photoelectrode with the ELs is obtained by calculation from the variations in light intensity.

Figure 8 presents the light intensity variations of the photoelectrode with and without the EL. Under one sun illumination of $1000 \mathrm{~W} / \mathrm{m}^{2}$ (AM 1.5), the photoelectrode without the EL yielded an intensity of $831 \mathrm{~W} / \mathrm{m}^{2}$ on the detector as the D-NTF. Depositing one EL on the FTO glass substrate of the photoelectrode increased intensity to $958 \mathrm{~W} / \mathrm{m}^{2}$ and light-harvesting efficiency by $15.28 \%$. When the number of ELs was increased to two, intensity increased to $1086 \mathrm{~W} / \mathrm{m}^{2}$, and light-harvesting efficiency increased by $30.69 \%$. As noted above, incorporating an EL effectively directed light toward the D-NTF of the photoelectrode, which enhanced light-harvesting efficiency.

\section{Preparation of DSSC}

The photoelectrode was fabricated as follows. First, an acetic solution was prepared by mixing $2 \mathrm{~g}$ citric acid, $0.08 \mathrm{~mL}$ acetylacetone, $0.2 \mathrm{~mL}$ Triton X-100, and $0.2 \mathrm{~mL}$ tetrabutyl orthotitanate in $3 \mathrm{~mL}$ purified water and stirring for $30 \mathrm{~min}$. Then, a $\mathrm{TiO}_{2}$ paste was prepared by mixing $1 \mathrm{~g} \mathrm{TiO}_{2}$ nanopowder (P25, Degussa) with acetic solution and stirring for $1 \mathrm{~h}$. The nanoporous $\mathrm{TiO}_{2}$ film was obtained by coating an FTO glass substrate (Hartford Glass Co., USA, fluorinedoped $\mathrm{SnO}_{2}$ overlayer, sheet resistance: $7 \Omega /$ sq) with $\mathrm{TiO}_{2}$ paste using a doctor-blade method and then sintering at $450^{\circ} \mathrm{C}$ for $30 \mathrm{~min}$. After sintering, measurement of the nanoporous $\mathrm{TiO}_{2}$ film with an Alpha-Step profilometer revealed 


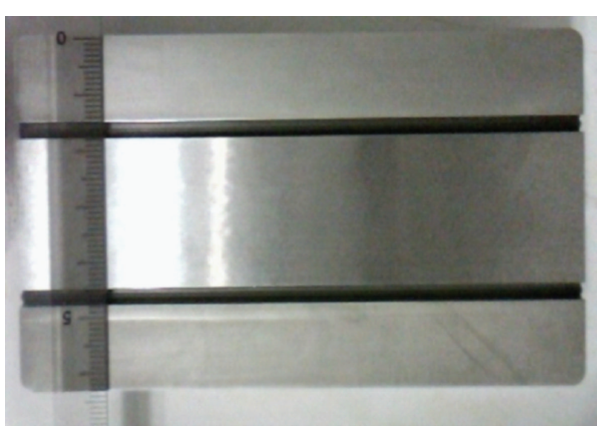

(a)

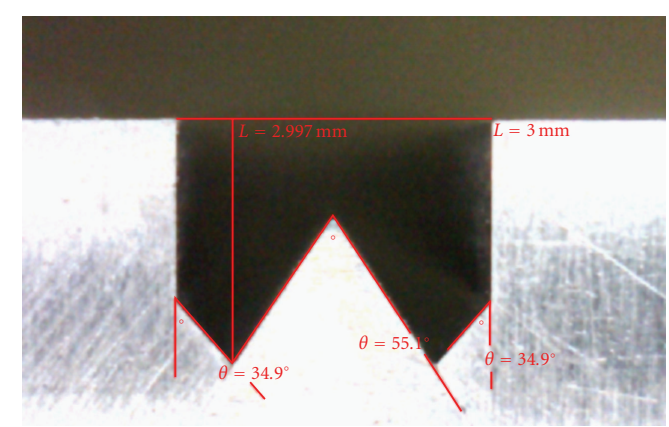

(b)

Figure 5: Photographs of top (a) and cross-section (b) of EL mold.

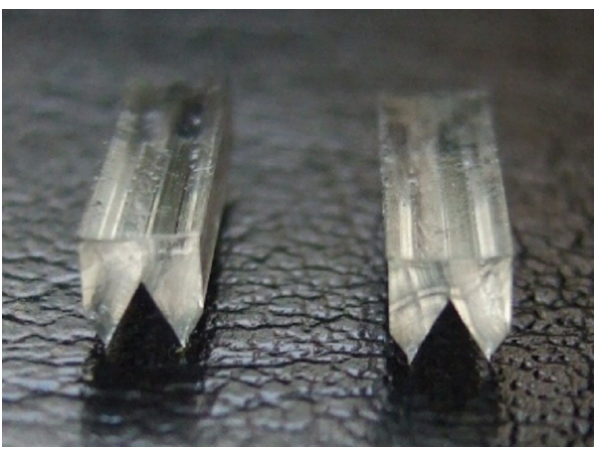

Figure 6: Photograph of ELs.

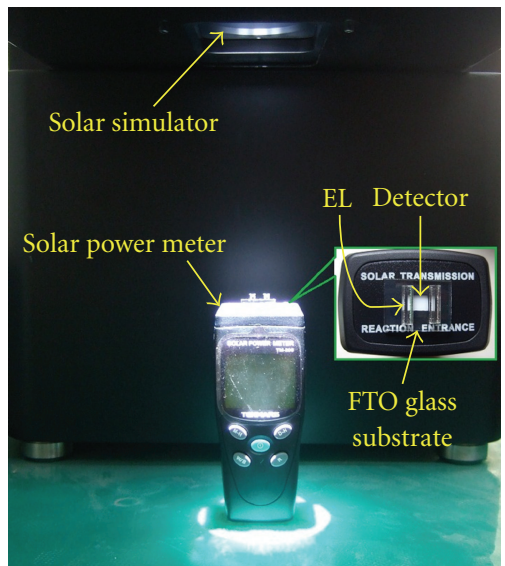

Figure 7: Experimental system for investigating light-harvesting efficiency.

a thickness of approximately $15 \mu \mathrm{m}$. The active area was 0.5 $\times 0.5 \mathrm{~cm}^{2}$.

The photoelectrode was then soaked in a dye solution of $0.3 \mathrm{mM}$ N719 dye in ethanol for $24 \mathrm{~h}$ at room temperature. The photoelectrode and the Pt counterelectrode were separated using a $60 \mu \mathrm{m}$-thick (before melting) surlyn polymer foil (Solaronix) as a sealing spacer. Sealing was performed by keeping the structure in a hot-press at $100^{\circ} \mathrm{C}$ for a few seconds. The final step of the DSSC preparation

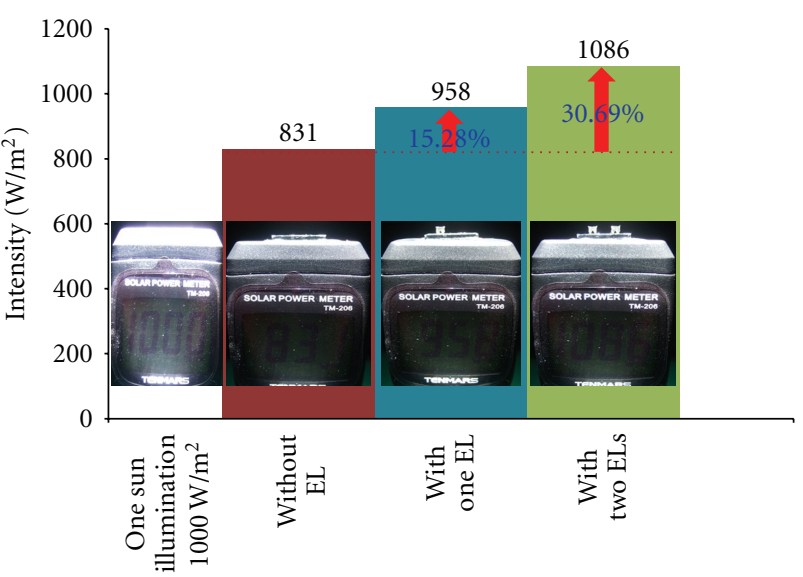

FIGURE 8: Variations in light intensity of photoelectrode with and without EL under one sun illumination of $1000 \mathrm{~W} / \mathrm{m}^{2}$ (AM 1.5).

was adding an electrolyte solution of $0.05 \mathrm{M}$ iodine, $0.1 \mathrm{M}$ lithium iodide, $0.5 \mathrm{M}$ 4-tert-butylpyridine (TBP), and $0.3 \mathrm{M}$ 1,2-dimethyl-3-propylimidazolium iodide (DMPII) in 3methoxy propionitrile (MPN) into the cell gap.

\section{Photovoltaic Measurements of DSSC with and without $\mathrm{EL}$}

To study the difference between the performance of DSSC with and without the EL, one EL and two ELs were attached to the exterior of the DSSC photoelectrode, respectively, as shown in Figure 9. The photovoltaic performance of DSSC was measured using a computer-controlled digital source meter (Keithley, Model: 2400) with an external bias applied to the cell under one sun illumination with a power density of $1000 \mathrm{~W} / \mathrm{m}^{2}$ (AM 1.5 simulated solar radiation). Figure 10 plots photocurrent density versus voltage for DSSC with and without the EL. Table 2 presents the corresponding photovoltaic performance. Under one sun illumination of $1000 \mathrm{~W} / \mathrm{m}^{2}$ (AM 1.5), the DSSC without the EL had an open-circuit voltage $\left(V_{\mathrm{oc}}\right)$ of $0.685 \mathrm{~V}$, a short-circuit current density $\left(J_{\mathrm{sc}}\right)$ of $6.90 \mathrm{~mA} / \mathrm{cm}^{2}$, a fill factor $(\mathrm{FF})$ of 0.591 , and a power conversion efficiency $(\eta)$ of $2.79 \%$. One EL improved the $J_{\mathrm{sc}}$ and $\eta$ of the DSSC to $8.04 \mathrm{~mA} / \mathrm{cm}^{2}$ and $3.16 \%$, which 


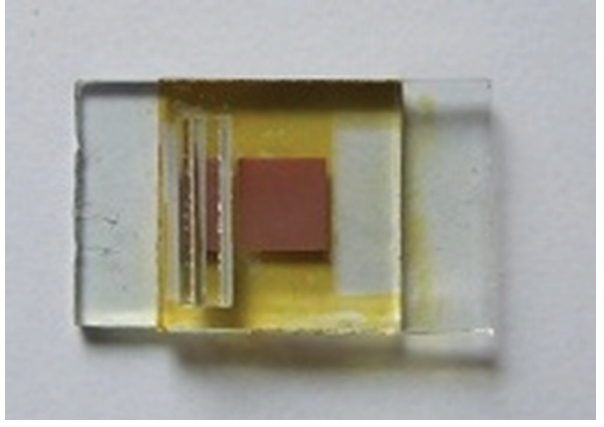

(a)

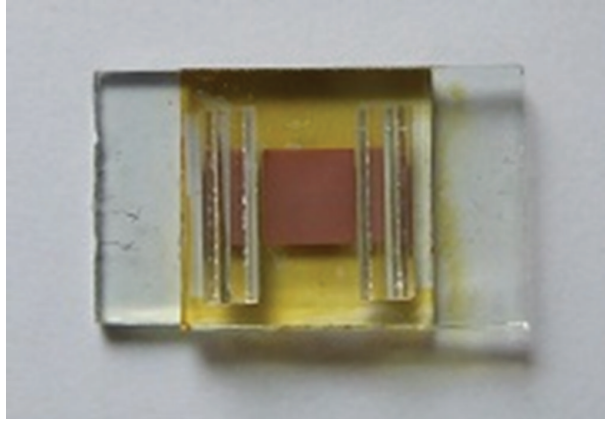

(b)

FIgURE 9: Photographs of DSSC with one EL (a) and two ELs (b).

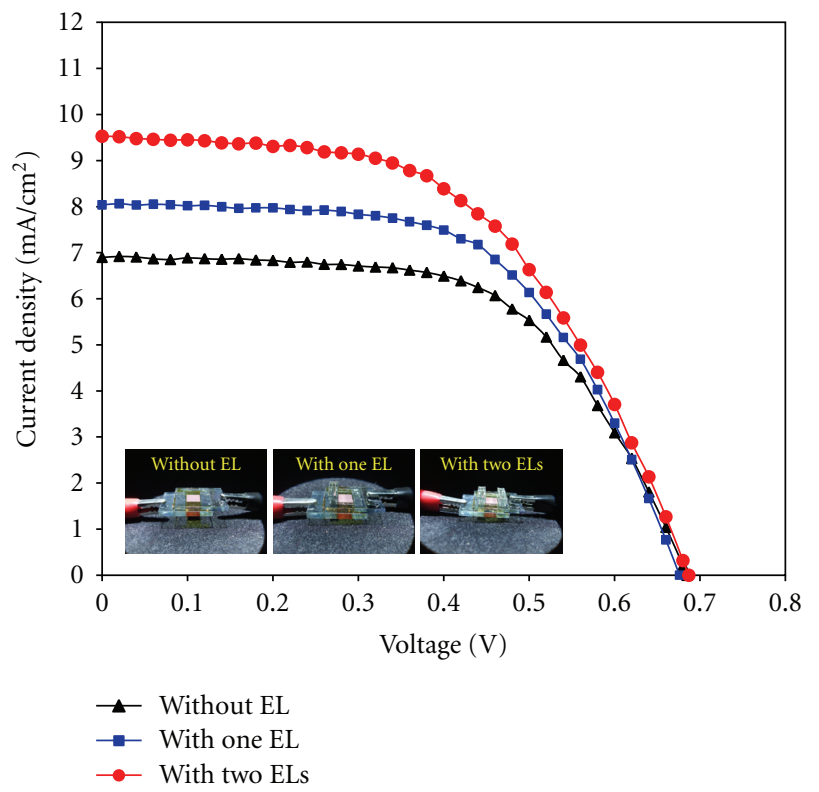

FIGURe 10: Photocurrent density-voltage curves of DSSC without EL (black triangle), with one EL (blue square), and with two ELs (red circle).

were improvements of $16.52 \%$ and $13.26 \%$, respectively. Two ELs increased $J_{\mathrm{sc}}$ and $\eta$ to $9.53 \mathrm{~mA} / \mathrm{cm}^{2}$ and $3.49 \%$, which were increases of $38.12 \%$ and $25.09 \%$, respectively. The lightharvesting efficiency increased with the photocurrent density and the power conversion efficiency. The increase in lightharvesting efficiency had a strong effect on the DSSC photocurrent density.

\section{Conclusion}

In summary, a transparent external lightguide (EL) was designed to improve the light-harvesting efficiency of a dyesensitized solar cell (DSSC). DSSCs with and without the EL were experimentally compared. The results indicate that the EL effectively directed light on the dye-covered nanoporous $\mathrm{TiO}_{2}$ film (D-NTF) of the photoelectrode, which enhanced light-harvesting efficiency, photocurrent density, and power conversion efficiency in the DSSC. Additionally, experimental results indicate that although the EL is applicable to low-efficiency, cost-effective DSSCs, its application to high-efficiency DSSCs is not limited. In future studies, the authors will consider potential applications of the EL in high-efficiency DSSCs, grid-type DSSCs, and other solar cell devices.

\section{Acknowledgment}

The authors would like to thank the National Science Council of Taiwan, for financially supporting this research under Contract no. NSC 99-2221-E-110-020.

\section{References}

[1] R. D. McConnell, "Assessment of the dye-sensitized solar cell," Renewable and Sustainable Energy Reviews, vol. 6, no. 3, pp. 273-295, 2002.

[2] G. Marsh, "Can dye sensitised cells deliver low-cost PV?" Renewable Energy Focus, vol. 9, no. 5, pp. 58-62, 2008.

[3] P. Wang, Ć. Klein, R. Humphry-Baker, S. M. Zakeeruddin, and M. Grätzel, "Stable $\geq 8 \%$ efficient nanocrystalline dye-sensitized solar cell based on an electrolyte of low volatility," Applied Physics Letters, vol. 86, no. 12, Article ID 123508, pp. 1-3, 2005.

[4] T. Oda, S. Tanaka, and S. Hayase, "Differences in characteristics of dye-sensitized solar cells containing acetonitrile and ionic liquid-based electrolytes studied using a novel model," Solar Energy Materials and Solar Cells, vol. 90, no. 16, pp. 2696-2709, 2006.

[5] A. Hauch and A. Georg, "Diffusion in the electrolyte and charge-transfer reaction at the platinum electrode in dye-sensitized solar cells," Electrochimica Acta, vol. 46, no. 22, pp. 3457-3466, 2001.

[6] L. Bay, K. West, B. Winther-Jensen, and T. Jacobsen, "Electrochemical reaction rates in a dye-sensitised solar cell-The iodide/tri-iodide redox system," Solar Energy Materials and Solar Cells, vol. 90, no. 3, pp. 341-351, 2006.

[7] X. Fang, T. Ma, G. Guan, M. Akiyama, T. Kida, and E. Abe, "Effect of the thickness of the Pt film coated on a counter electrode on the performance of a dye-sensitized solar cell," Journal of Electroanalytical Chemistry, vol. 570, no. 2, pp. 257263, 2004.

[8] K. Okada, H. Matsui, T. Kawashima, T. Ezure, and N. Tanabe, "100 mm × $100 \mathrm{~mm}$ large-sized dye sensitized solar cells," 
Journal of Photochemistry and Photobiology A, vol. 164, no. 13, pp. 193-198, 2004.

[9] W. Kubo, A. Sakamoto, T. Kitamura, Y. Wada, and S. Yanagida, "Dye-sensitized solar cells: improvement of spectral response by tandem structure," Journal of Photochemistry and Photobiology A, vol. 164, no. 1-3, pp. 33-39, 2004.

[10] W. J. Lee, E. Ramasamy, D. Y. Lee, and J. S. Song, "Grid type dye-sensitized solar cell module with carbon counter electrode," Journal of Photochemistry and Photobiology A, vol. 194, no. 1, pp. 27-30, 2008.

[11] E. Ramasamy, W. J. Lee, D. Y. Lee, and J. S. Song, "Portable, parallel grid dye-sensitized solar cell module prepared by screen printing," Journal of Power Sources, vol. 165, no. 1, pp. 446-449, 2007. 


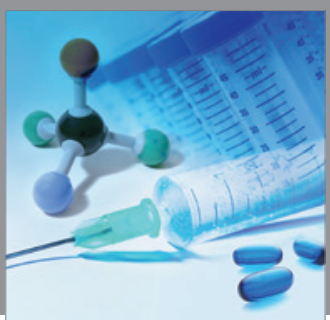

International Journal of

Medicinal Chemistry

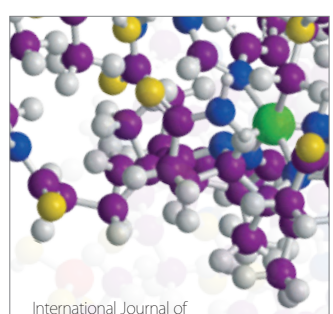

Carbohydrate Chemistry

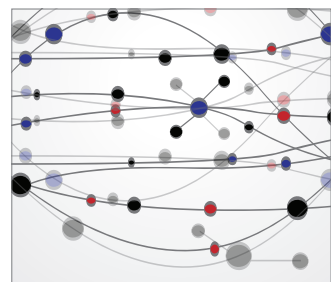

The Scientific World Journal
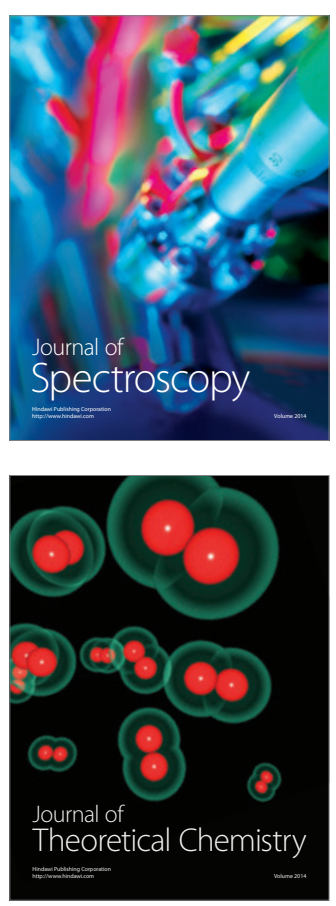
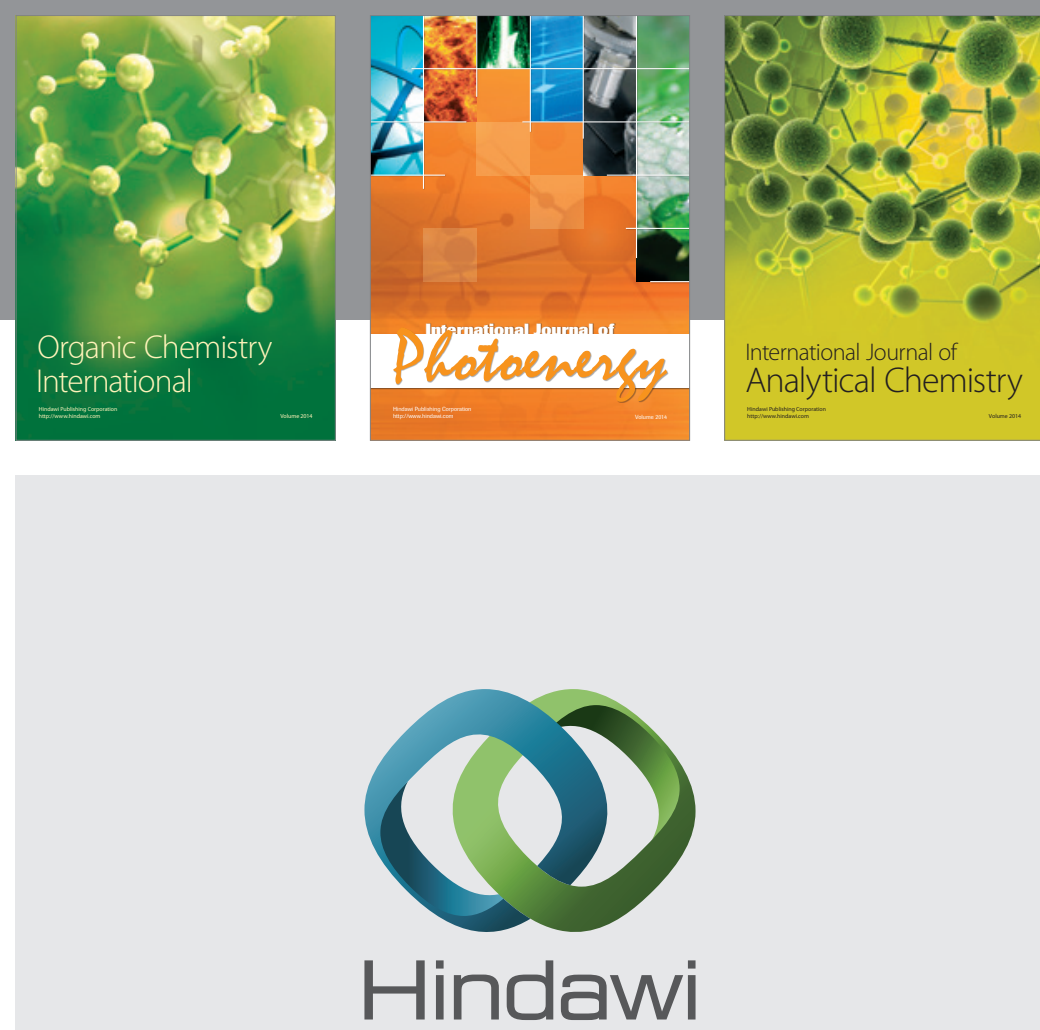

Submit your manuscripts at

http://www.hindawi.com
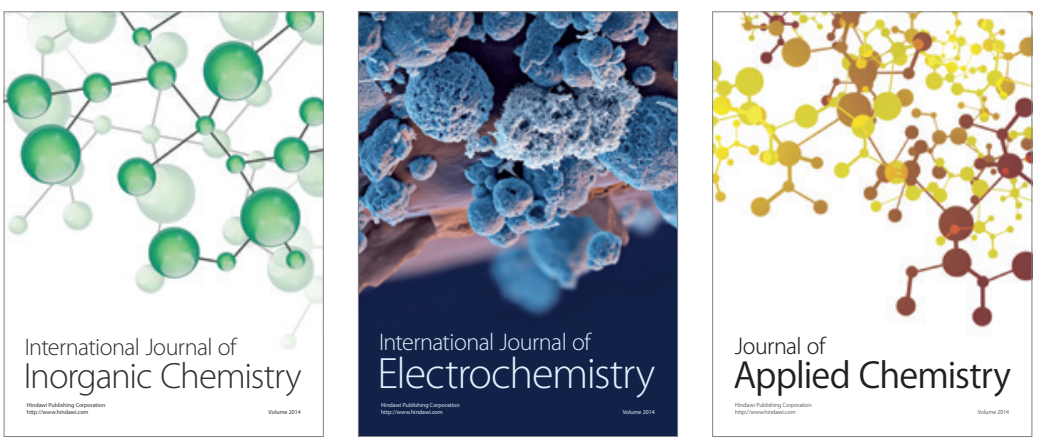

Journal of

Applied Chemistry
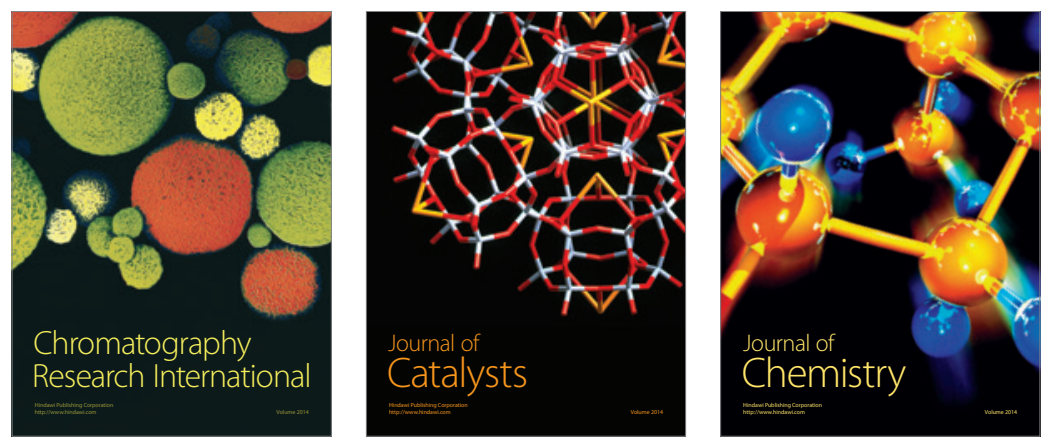
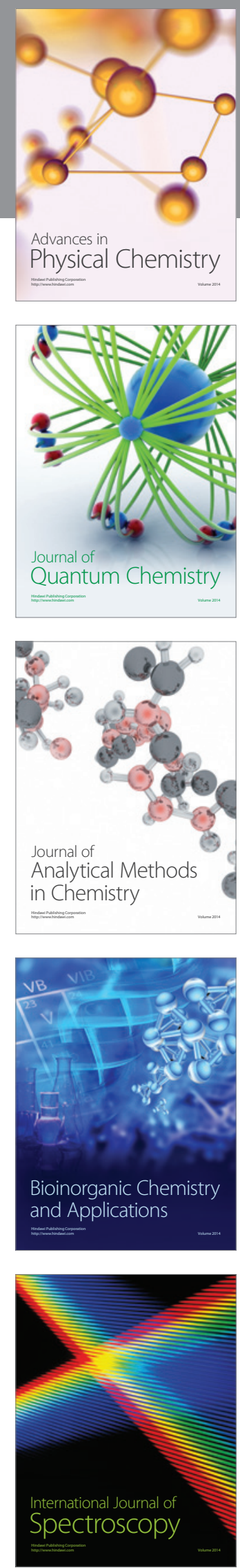